\title{
Coating of Sub-Micrometric Keratin Fibers on Titanium Substrates: A Successful Strategy for Stimulating Adhesion and Alignment of Fibroblasts and Reducing Bacterial Contamination
}

\author{
Sara Ferraris 1,*, Vincenzo Guarino ${ }^{2}$, Andrea Cochis ${ }^{3,4}$, Alessio Varesano 5, Iriczalli Cruz Maya 2,6, \\ Claudia Vineis ${ }^{5}$, Lia Rimondini ${ }^{3,4}$ and Silvia Spriano ${ }^{1}$ \\ 1 Department of Applied Science and Technology, Politecnico di Torino, 10129 Torino, Italy; \\ silvia.spriano@polito.it \\ 2 CNR-IPCB, Institute of Polymers, Composites and Biomaterials, 80125 Napoli, Italy; \\ vincenzo.guarino@cnr.it (V.G.); cdiriczalli@gmail.com (I.C.M.) \\ 3 Department of Health Sciences, Università del Piemonte Orientale, 28100 Novara, Italy; \\ andrea.cochis@med.uniupo.it (A.C.); lia.rimondini@med.uniupo.it (L.R.) \\ 4 Center for Translational Research on Autoimmune \& Allergic Diseases-CAAD, 28100 Novara, Italy \\ 5 CNR-ISMAC, Institute for Macromolecular Studies, 13900 Biella, Italy; a.varesano@bi.ismac.cnr.it (A.V.); \\ c.vineis@bi.ismac.cnr.it (C.V.) \\ 6 Department of Chemical, Materials and Industrial Production Engineering, University of Naples Federico \\ II, Naples, Italy \\ * Correspondence: sara.ferraris@polito.it
}

Received: 15 February 2019; Accepted: 19 February 2019; Published: 28 February 2019

\begin{abstract}
Coatings are a versatile tool for modulation of the biological response of biomaterials; in particular, the use of biopolymers as coating material may improve cell interactions and tissue adhesion. Among others, keratin is a natural protein able to stimulate fibroblast cells effectively and has the ability to bind metal ions. Coatings of keratin fibers onto titanium substrates can improve soft tissue adhesion, eventually coupling topographical (contact guidance) and chemical stimulus through the alignment of the fibers along sub-micrometric grooves of the substrate. Submicrometric keratin fibers were obtained by electrospinning both in random and oriented arrangements (though a rotating collector); in addition, antibacterial properties were added by enrichment of the coating with silver ions. This type of coating can be of interest in transmucosal dental implants, where perimplantitis is often due to infection (biofilm formation) and disease worsening is due to inflammation of the surrounding soft tissue, which is guided by fibroblasts. Keratin fibres coatings were prepared and characterized by means of Field Emission scanning electron microscopy (FESEM), X-ray photoelectron spectroscopy (XPS), cell (gingival fibroblasts) and bacteria (S. aureus) culture tests. FESEM observations demonstrated the possibility to deposit keratin fibres onto titanium substrates in random or oriented arrangements effectively. Keratin fibres were able to increase fibroblast adhesion and proliferation. On randomly deposited keratin fibres, fibroblast cells were significantly biologically stimulated and showed high adhesion and proliferation, but not orientation ability; on the other hand, aligned keratin fibres on a grooved substrate were able to stimulate cells both from the topographical (orientation) and biological standpoint. Finally, Ag-doped keratin fibres coatings were able to reduce S. aureus adhesion significantly, maintaining high biocompatibility. Considering these results, keratin submicrometric fibres coatings are a promising strategy for stimulating fibroblasts and reducing bacterial contamination.
\end{abstract}

Keywords: titanium; keratin; electrospinning; fibroblasts; antibacterial 


\section{Introduction}

Effective soft tissue bonding and prevention of bacterial contamination are key issues in several implants such as trans-skin orthopedic and trans-mucosal dental implants [1,2]. Despite of a wide research on surface modification of titanium surfaces for bone contact applications, few research works focus on innovative surface treatments and coatings able to support soft tissue adhesion and limit bacterial contamination.

Fibroblasts, the main constituent of soft tissues, are generally rugo-phobic cells but they can align along specific directions (e.g., grooves) according to the well-known contact guidance phenomenon [3,4]. Moreover this kind of cell can be effective stimulated by biochemical factors such as keratin protein [5]. Following this rationale surfaces with oriented patterns and keratin enrichment can be suitable for the stimulation of fibroblast cells and the guidance of soft tissue adhesion in a healthy and effective way.

In addition, it is widely reported in literature that keratin has a high affinity for metal ions [6]. This ability is usually exploited in the water treatment field [7], but it can be also considered for the introduction of antibacterial properties in keratin-based materials.

Among different antibacterial metal ions, silver has been selected because it is well known for its broad spectrum antibacterial activity (based on a multiple mechanism of action), moreover it is associated with a low risk of resistance development, differently from antibiotics [8-10].

In the present research work, coatings of sub-micrometric keratin fibres were obtained onto commercially pure titanium substrates by electrospinning. Keratin was obtained from discarded wool following a green approach, which supports the employment of abundant local byproducts for the production of high added value coating intended for biomedical applications. Randomly oriented or aligned sub-micrometric fibres were deposited using stationary or rotating collectors respectively. Finally, deposited coatings were enriched with silver ions in order to confer also antibacterial functionalities.

\section{Materials and Methods}

Commercially pure titanium disks (ASTM B348 [11], Gr2, Titanium Consulting and Trading, 2 $\mathrm{mm}$ thick and $10 \mathrm{~mm}$ in diameter) and foils (Titanium foil $0.025 \mathrm{~mm}, 99.94 \%$, Alfa Aesar, Haverhill, MA, USA) were used as substrates for keratin sub-micrometric fibres deposition with stationary and rotating collectors, respectively $[12,13]$.

Keratin was extracted from discarded wool by sulphitolysis with sodium metabisulphite and freeze dried, as previously described [12].

Randomly oriented keratin sub-micrometric fibres were deposited onto titanium substrates from keratin solutions 15\% w/w in formic acid (reagent grade, 95\%, Sigma-Aldrich, St. Louis, MO, USA) by means of a typical electrospinning setup composed by a plastic syringe, a highprecision syringe pump (KDS200, KD Scientific, Inc., Holliston, MA, USA) and a generator (SL50, Spellman High Voltage Electronics Corp., Hauppauge, NY, USA), which supplied a voltage of $25 \mathrm{kV}$. A stainless steel plate was used as collector and titanium disks substrates were attached to it for coating [12].

Aligned keratin sub-micrometric fibres were deposited onto titanium foils from keratin solutions $10 \% w / v$ in 1,1,1,3,3,3-hexafluoro-2-propanol (HFIP, Sigma-Aldrich, Milan, Italy) by means of a commercial electrospinning system (NANON 01, MECC, Fukuoka, Japan) equipped with a rotating collector and 18 Ga needle[13].

Varying the deposition conditions (time, temperature, humidity) different amounts of submicrometric fibers was obtained onto titanium substrates, with consequently different surface coverage degrees [12,13]. Occasionally some beads were also formed [12].

Coated specimens were thermally treated $2 \mathrm{~h}$ at $180^{\circ} \mathrm{C}$ in order to stabilize the fibres [12-14].

Keratin ability to bind metal ions [6] was exploited in order to confer antibacterial activity to coated surfaces, in this work. Titanium samples, coated with randomly oriented sub- 
micrometric keratin fibres, were soaked in silver nitrate aqueous solutions $(0.01,0.05$ and $0.1 \mathrm{M})$ for $5 \mathrm{~h}$ at room temperature, as described in [15].

Surface topography of coated samples was investigated by means of field emission scanning electron microscopy equipped with energy dispersive spectroscopy (FESEM-EDS SUPRATM 40, Zeiss and Merlin Gemini Zeiss, Oerzen, Germany).

Cytocompatibility and cellular alignment were evaluated with human primary gingival fibroblasts (HGFs) from normal human gingiva, as representative cells for the possible final application of the proposed coatings and as previously reported [12]. Cells were seeded in a defined number $\left(2 \times 10^{4}\right.$ cells/specimen) directly onto samples' surface and cultivated up to $48 \mathrm{~h}$ after that cells morphology was evaluated by fluorescence imaging, after fixation with paraformaldehyde and staining with phalloidin and DAPI to visualize cytoskeleton f-actins filaments and nuclei, respectively.

Antibacterial activity was evaluated against Staphylococcus aureus (ATCC 25923). Coated samples were covered with $1 \mathrm{~mL}$ of LB medium containing $1 \times 10^{5}$ cells $/ \mathrm{ml}$ and incubated for $90 \mathrm{~min}$ at $37^{\circ} \mathrm{C}$ under agitation $(120 \mathrm{rpm})$. Supernatants were then extracted and samples gently washed 3 times (with PBS) to remove non-adherent cells. Each specimen was rinsed with $1 \mathrm{ml}$ of fresh LB medium and plate incubated $\left(37^{\circ} \mathrm{C}\right)$ up to $24 \mathrm{~h}$ for biofilm cultivation [12]. To assess the growth capacity of the bacterial strains in direct contact, bacterial viability was evaluated by colorimetric metabolic assay (XTT, Sigma-Aldrich). Moreover, the count of colony forming units (CFU) was also performed, as described in [15]; biofilm morphology was visually checked by SEM.

\section{Results and Discussion}

FESEM observations of randomly oriented and aligned sub-micrometric keratin fibres are reported in Figure 1.
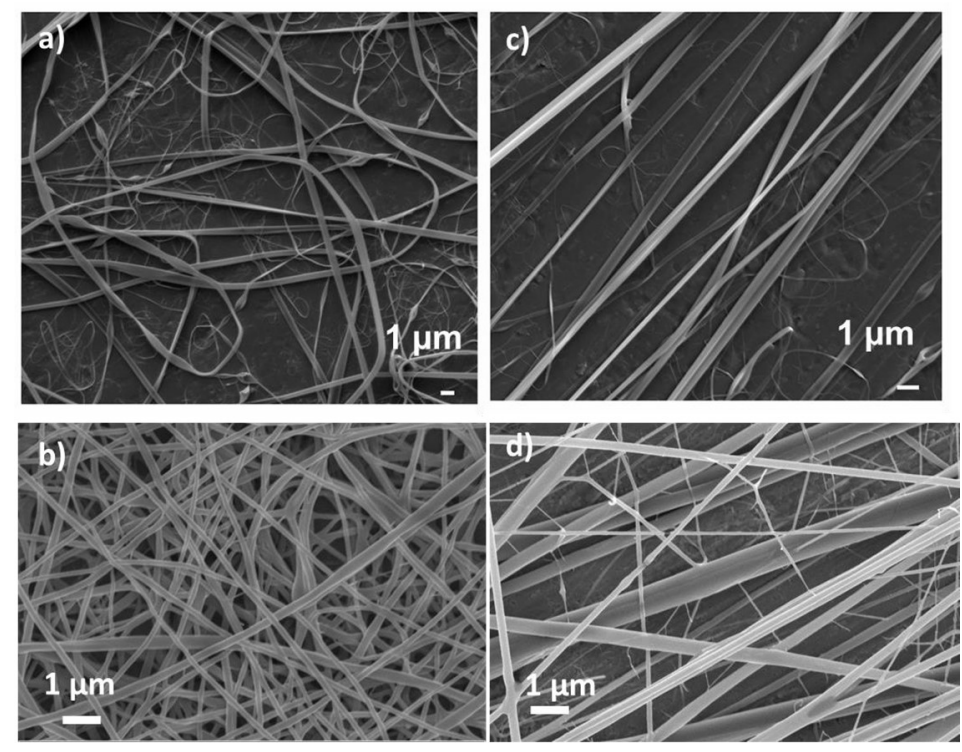

Figure 1. FESEM micrographs of keratin sub-micrometric fibres: (a) low density randomly oriented; (b) high density randomly oriented; (c) low density aligned; and (d) high density aligned.

Depending on the process parameters, different coating density can be obtained. Different degrees of substrate coverage can be of interest in order to combine, for example, substrate topographical properties with biochemical stimuli of keratin fibres, as reported by the authors in $[12,13]$.

Moreover, mats of randomly oriented fibers or aligned fibers can be obtained by changing the type of collector (stationary or rotating collector, respectively). The second typology of fibres can be of interest, again, with the final aim to combine biochemical signals from keratin with 
topographical stimulation of substrate topography and fibres direction. In fact, fibroblast cells are highly sensitive to contact guidance [3,4] as well as to keratin biochemistry [5].

Biological tests confirm this hypothesis. HGF show to align along the fibres direction in presence of a defined direction of keratin deposition (Figure 2), thus confirming keratin fibers ability to drive fibroblasts adhesion and spread as also previously demonstrated by the Authors [12,13].

a)

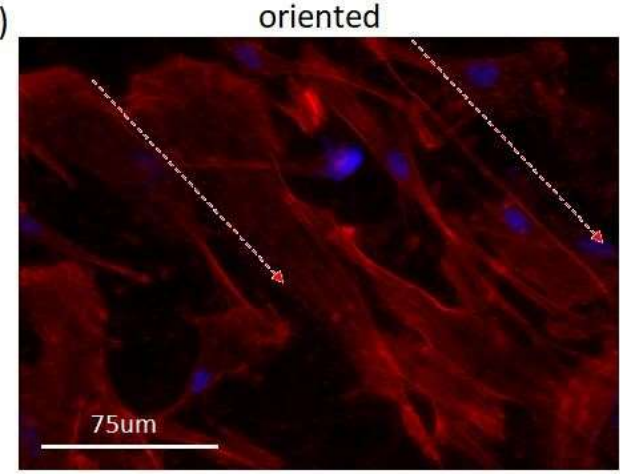

b)

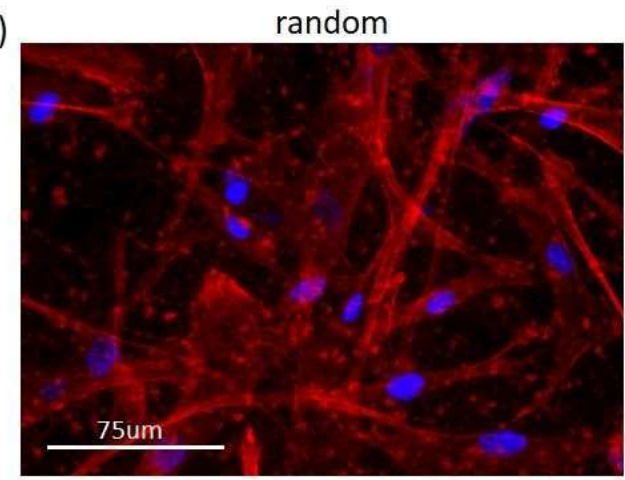

Figure 2. Cells alignment. The oriented keratin coating was able to influence cells spread towards it (a) in comparison with the random one (b). Bar scale $=75 \mu \mathrm{m}$; Mag. $=20 \times$.

FESEM images (100,000× magnifications) of silver doped keratin sub-micrometric fibers are reported in Figure 3: low keratin density samples in the first row and high keratin density samples in the second row. The concentration of silver in the treatment solution increase from left to right (from $0.001 \mathrm{M}$ to $0.1 \mathrm{M}$ ). On all the samples, keratin sub-micrometric fibers with few beads have been observed as a result of the electrospinning deposition, as previously reported by the authors [12]. As far as the low keratin density samples are concerned, EDS analyses on a keratin fibre or bead reveal the presence of silver together with the main constituents of keratin (i.e., C, N, $\mathrm{O}, \mathrm{S})$. Sub-micrometric precipitates are visible on the uncoated zones of the metallic substrates (dotted arrows in Figure $3 a-c)$; EDS analyses on the precipitates (Figure 3i) underline that they are constituted mainly by silver. Looking at the high density keratin samples (Figure $3 \mathrm{~d}-\mathrm{f}$ ), it can be noted that the titanium substrate is hindered by the high density keratin layer, so it is almost impossible to analyze separately fibres and beads as well as to observe eventual precipitates on the titanium substrates. EDS analysis on the sample area (Figure 31) evidences the presence of titanium, keratin constituents and silver.

It seems that in the case of high keratin density coating, almost all silver ions have been loaded onto keratin fibres, as previously reported by the authors [15]. On the other hand, low keratin density coating maintains a portion of titanium surface free from coating and in this case, silver has been loaded on the surface in two forms: into keratin fibres/beads and in the form of submicrometric precipitates on the metallic substrate. Since keratin is able to attract and retain metallic ions and titanium is able to reduce silver ions to metallic silver (due to its negative redox standard potential) $[6,7,16]$ it can be supposed that ionic silver has been introduced into keratin while metallic silver has been precipitated on the metallic substrate.

Antibacterial tests revealed the ability of all Ag-doped coated samples to reduce bacterial adhesion and biofilm formation (Figure 4). In fact, $S$. aureus viability was significantly reduced by comparing Ag-doped specimens and keratin control ones (Figure $4 \mathrm{a}, p<0.05$, indicated by $\S$ ) as well as the number of viable colonies was significantly lowered (Figure $4 b, p<0.05$, indicated by $\S$ ). Finally, SEM images confirmed that bacteria in contact with Ag ions grafted onto specimens' surface were dead as they presented typical membrane damages due to Ag ions accumulation (Figure 4c). 

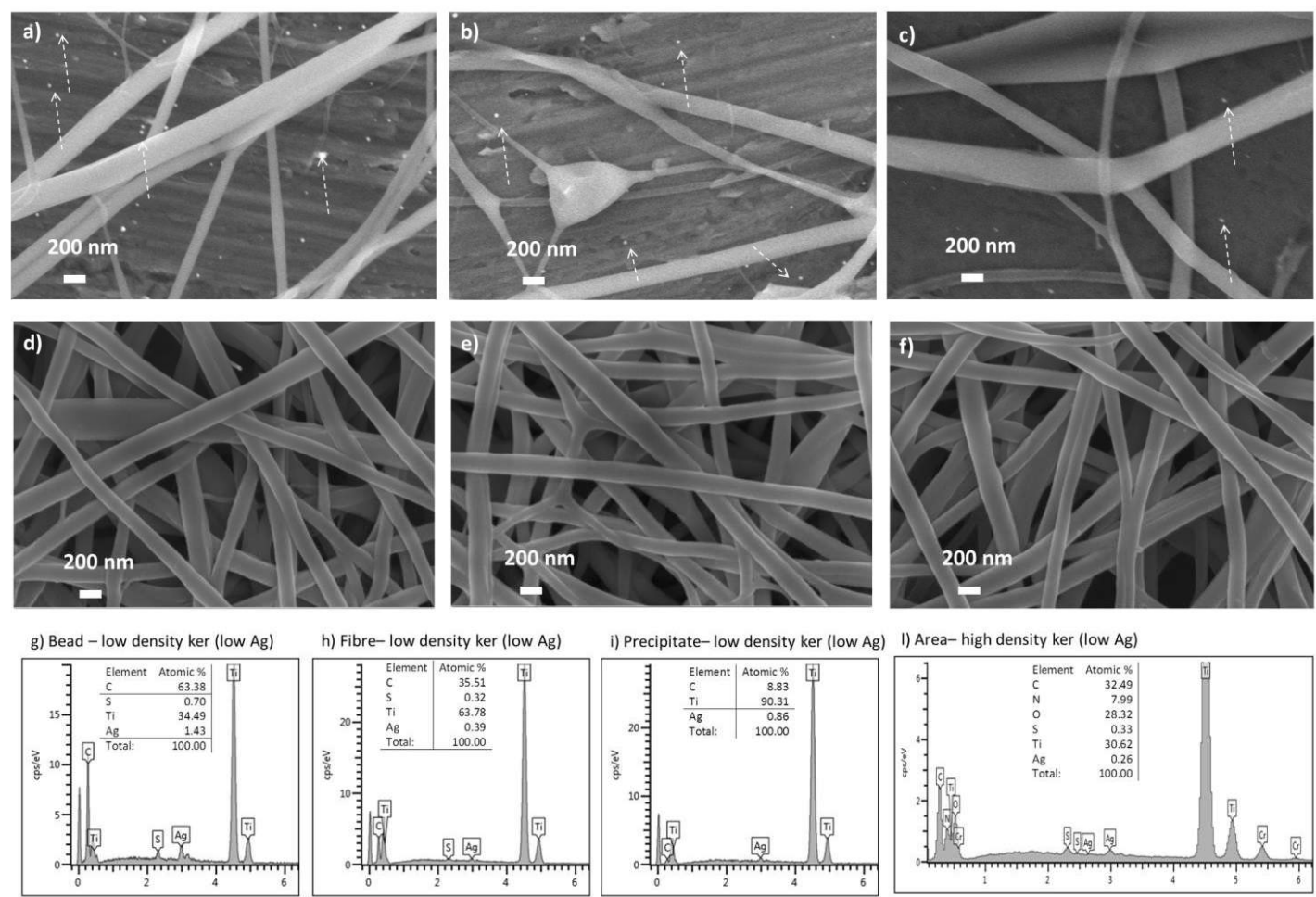

Figure 3. FESEM images of low keratin density samples $\mathrm{Ag}$ doped in $0.01 \mathrm{M} \mathrm{AgNO}_{3}(\mathbf{a}), 0.05 \mathrm{M}$ $\mathrm{AgNO}_{3}(\mathbf{b})$ and $0.1 \mathrm{M} \mathrm{AgNO}_{3}(\mathbf{c})$; and high density keratin samples Ag doped in $0.01 \mathrm{M} \mathrm{AgNO}_{3}(\mathbf{d})$, $0.05 \mathrm{M} \mathrm{AgNO}_{3}$ (e) and $0.1 \mathrm{M} \mathrm{AgNO}_{3}$ (f). EDS analyses on a keratin bead (low density keratin samples $\mathrm{Ag}$ doped in $\mathrm{AgNO}_{3} 0.01 \mathrm{M}$ (g); EDS analysis on a keratin fibre (low density keratin samples $\mathrm{Ag}$ doped in $\mathrm{AgNO}_{3} 0.01 \mathrm{M}$ (h); EDS analysis on a precipitate (low density keratin samples $\mathrm{Ag}$ doped in $\mathrm{AgNO}_{3} 0.01 \mathrm{M}(\mathbf{i})$; and EDS analysis on the area of a high density keratin sample $\mathrm{Ag}$ doped in $\mathrm{AgNO}_{3} 0.01 \mathrm{M}(\mathbf{l})$.
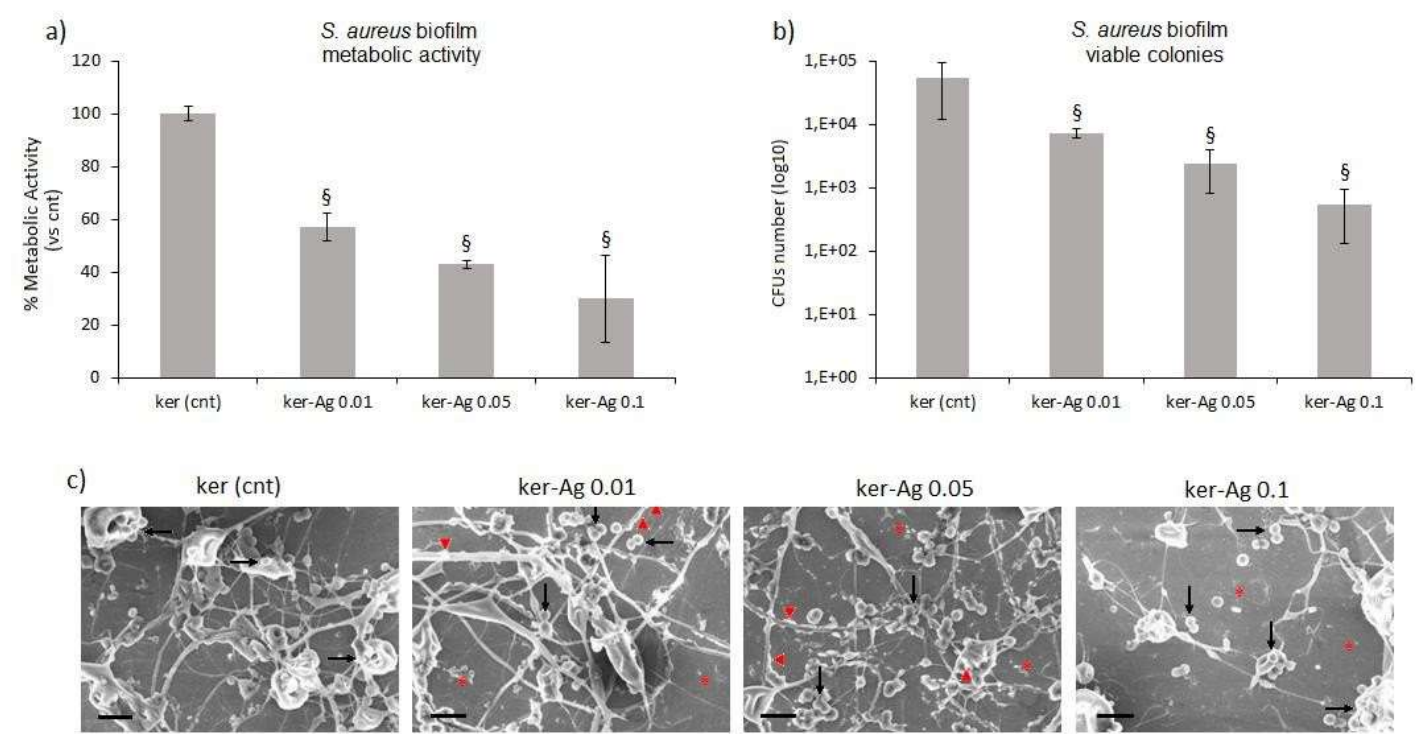

Figure 4. Antibacterial activity. The Ag-doping determined a significant reduction of $S$. aureus metabolic activity (a) and viable colonies (b) in comparison with bare keratin controls $(p<0.05$, indicated by §). SEM images (c) showed the typical membrane damages due to Ag ions. Bars represent means and standard deviations; SEM bar scale $=2 \mathrm{~mm}$, Mag. $=10 \mathrm{k} \times$ ). 
This observation, in addition to what previously discussed about topography, underlines that the possibility to tailor keratin coverage of titanium surfaces give the possibility to select the different stimuli to be administered to cells (topographical or biochemical), but also to select, different antibacterial strategies, (such as silver ions or nanoparticles) and silver loading ability.

All the Ag-doped coatings maintain complete biocompatibility for HGF cells, as reported by the authors in [15].

The rationale and main findings of the research is summarized in Figure 5.

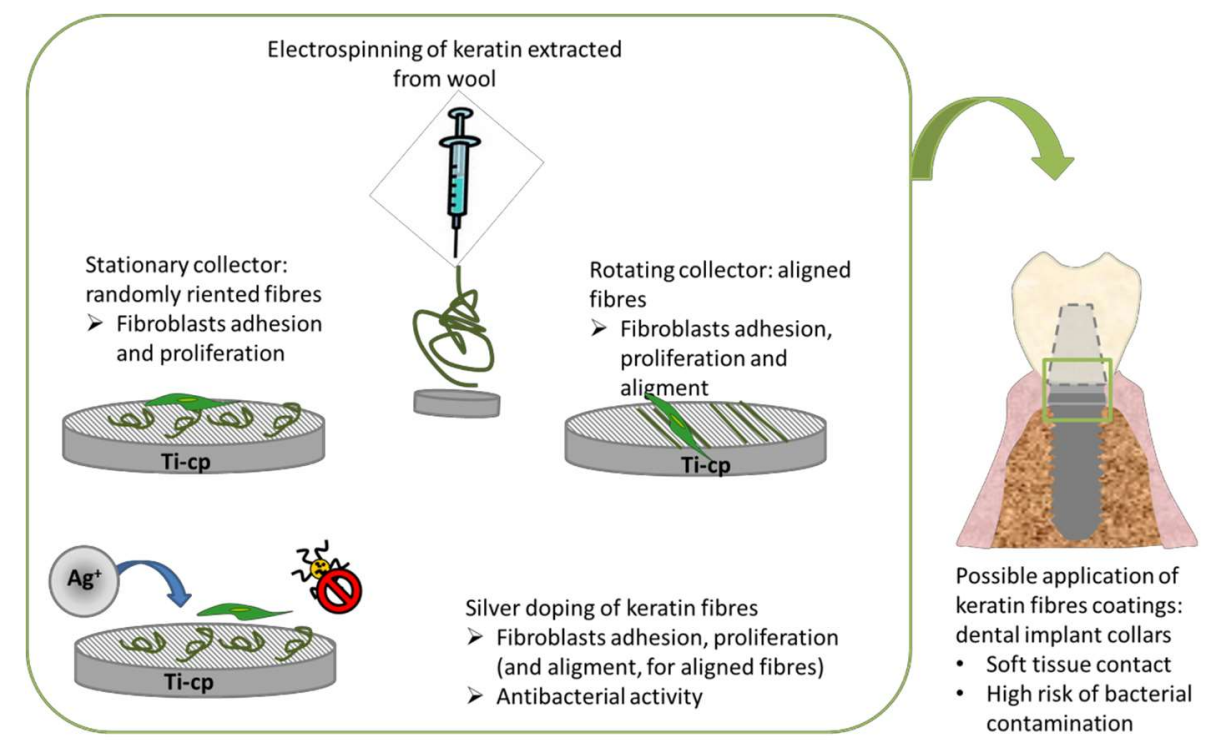

Figure 5. Summary of research rationale and main findings.

Sub-micrometric keratin fibres coating have been deposited onto titanium substrates by electrospinning with random or oriented disposition. The so-produced coatings resulted perfectly biocompatible and able to support HGF adhesion and proliferation, as well as alignment in case of sub-micrometric keratin fibres deposited with a preferential direction. Silver enrichment of modified surfaces depends on the coating density and, in all cases, allows the production of biocompatible coatings able to reduce bacterial adhesion. In conclusion the here described coatings can match the main requirements of titanium surfaces facing soft tissues, such as transmucosal dental implants, which main problems are poor gum sealing and bacterial contamination.

\section{Conclusions}

Keratin obtained by discarded wool by a green approach was successfully used for the preparation of high added value coatings intended for biomedical applications. Sub-micrometric keratin nanofibers were obtained with random orientation on plane Ti-disks by means of electrospinning deposition with stationary collector while oriented fibres were produced by means of the application of a rotating collector. The ability of keratin to bind metal ions was exploited for fibres enrichment with antibacterial silver ions.

Conflicts of Interest: The authors declare no conflict of interest.

\section{References}

1. Campoccia, D.; Montanaro, L.; Arciola, C.R.A. Review of the biomaterials technologies for infectionresistant surfaces. Biomaterials 2013, 34, 8533-8554.

2. Wang, X.; Lu, T.; Wen, J.; Xu, L.; Zeng, D.; Wu, Q.; Cao, L.; Lin, S.; Liu, X.; Jiang, X. Selective responses of human gingival fibroblasts and bacteria on carbon fibre reinforced polyetheretherketone with multilevel nanostructured $\mathrm{TiO}_{2}$. Biomaterials 2016, 83, 207-218. 
3. Brunette, D.M. Principles of cell behavior on titanium surfaces and their application to implanted devices. In Titanium in Medicine: Material Science, Surface Science, Engineering, Biological Responses and Medical Applications; Brunette, D.M., Tengvall, P., Textor, M., Thomsen, P., Eds.; Springer-Verlag: Berlin/Heidelberg, Germany, 2001, pp. 485-512.

4. Jaeger, N.A.F.; Brunette, D.M. Production of microfabricated surfaces and their effects on cell behavior. In Titanium in Medicine: Material Science, Surface Science, Engineering, Biological Responses and Medical Applications; Brunette, D.M., Tengvall, P., Textor, M., Thomsen, P., Eds.; Springer-Verlag: Berlin/Heidelberg, Germany, 2001, pp. 343-374.

5. Aluigi, A.; Zoccola, M.; Vineis, C.; Tonin, C.; Ferrero, F.; Canetti, M. Study on the structure and properties of wool keratin regenerated from formic acid. Int. J. Biol. Macromol. 2007, 41, 266-273.

6. Aluigi, A.; Tonetti, C.; Vineis, C.; Tonin, C.; Mazzuchetti, G. Adsorption of copper(II) ions by keratin/PA6 blend nanofibers. Eur. Polym. J. 2011, 47, 1756-1764.

7. Aluigi, A.; Vineis, C.; Tonin, C.; Tonetti, C.; Varesano, A.; Mazzuchetti, G. Wool keratin-based nanofibres for active filtration of air and water. J. Biobased Mater. Bioenergy 2009, 3, 311-319.

8. Knetsch, M.L.W.; Koole, L.H. New strategies in the development of antimicrobial coatings: the example of increasing usage of silver and silver nanoparticles. Polymers 2011, 3, 340-366.

9. Lemire, J.A.; Harrison, J.J.; Turner, R.J. Antimicrobial activity of metals: Mechanisms, molecular targets and applications. Nat. Rev. 2013, 11, 371.

10. Ovington, L.G. The truth about silver. Ostomy/Wound Manag. 2004, 50,1S-10S.

11. ASTM B348-13 Standard Specification for Titanium and Titanium Alloy Bars and Billets; ASTM International, West Conshohocken, PA, USA, 2013.

12. Ferraris, S.; Truffa Giachet, F.; Miola, M.; Bertone, E.; Varesano, A.; Vineis, C.; Cochis, A.; Sorrentino, R.; Rimondini, L.; Spriano, S. Nanogrooves and keratin nanofibres on titanium surfaces aimed at driving gingival fibroblasts alignment and proliferation without increasing bacterial adhesion. Mater. Sci. Eng. C 2017, 76, 1-12.

13. Ferraris, S.; Guarino, V.; Cochis, A.; Varesano, A.; Maya, I.C.; Vineis, C.; Rimondini, L.; Spriano, S. Aligned keratin submicrometric-fibres for fibroblasts guidance onto nanogrooved titanium surfaces for transmucosal implants. Mater. Lett. 2018, 229, 1-4.

14. Aluigi, A.; Corbellini, A.; Rombaldoni, F.; Zoccola, M.; Canetti, M. Morphological and structural investigation of wool-derived keratin nanofibres crosslinked by thermal treatment. Int. J. Biol. Macromol. 2013, 57, 30-37.

15. Cochis, A.; Ferraris, S.; Sorrentino, R.; Azzimonti, B.; Novara, C.; Geobaldo, F.; Truffa Giachet, F.; Vineis, C.; Varesano, A.; Abdelgeliel, A.S.; Spriano, S.; Rimondini, L. Silver-doped keratin nanofibres preserve a titanium surface from biofilm contamination and favor soft-tissue healing. J. Mater. Chem. B 2017, 5, 83668377.

16. Ferraris, S.; Venturello, A.; Miola, M.; Cochis, A.; Rimondini, L.; Spriano, S. Antibacterial and bioactive nanostructured titanium surfaces for bone integration. Appl. Surf. Sci. 2014, 311, 279-291.

(C) 2019 by the authors. Licensee MDPI, Basel, Switzerland. This article is an open access article distributed under the terms and conditions of the Creative Commons Attribution (CC BY) license (http://creativecommons.org/licenses/by/4.0/). 